\title{
The Consistent Estimators for Homogeneous Gaussian Fields Statistical Structures
}

\author{
Z. Zerakidze, M. Patsasia. \\ Gori University, Gori, Georgia, Sukhumi University, Tbilisi, Georgia.
}

\begin{abstract}
The present theory of consistent estimators of the parameters of statistical structures of homogeneous Gaussian fields can be used, for example, in the reliability predication of different engineering designs. In the paper there are discussed Gaussian homogeneous fields statistical structures $\left\{E, S, \mu_{i}, i \in I\right\}$ in Banach space of measures. We prove necessary and sufficient conditions for existence of such estimators.
\end{abstract}

Keywords: consistent estimators, orthogonal, weakly separable, strongly separable statistical structures. Classification 62HO5, $62 \mathrm{H} 12$

\section{Introduction}

Let there is given $(E, S)$ measurable space and on this space there given $\left\{\mu_{i}, i \in I\right\}$ family of probability measures depended on $\mathrm{S}$, the I set of parameters.

Let bring some definition (see [1]-[10]).

Definition 1. A statistical structure is called object $\left\{E, S, \mu_{i}, i \in I\right\}$ where $i \in I$ some parameter associated with probability measure $\mu_{i}$, I set of parameters.

Definition 2. A statistical structure $\left\{E, S, \mu_{i}, i \in I\right\}$ is called orthogonal (singular) if $\mu_{i}$ and $\mu_{j}$ are orthogonal for each $i \neq j, \quad i \in I, \quad j \in I$.

Definition 3. A statistical structure $\left\{E, S, \mu_{i}, i \in I\right\}$ is called weakly separable if there exists family $\mathrm{S}$ measurable $\quad \operatorname{sets}\left\{X_{i}, i \in I\right\}$ such that relations are fulfilled:

$$
(\forall i)(\forall j)(i \in I \& j \in I) \Rightarrow \mu_{i}\left(X_{j}\right)=\left\{\begin{array}{l}
1, \text { if } i=j \\
0, \text { if } i \neq j
\end{array} .\right.
$$

Definition4. A statistical structure $\left\{E, S, \mu_{i}, i \in I\right\}$ is called strongly separable if there exists disjoint family Smeasurable sets $\left\{X_{i}, i \in I\right\}$ such that the relation are fulfilled. $\mu_{i}\left(X_{i}\right)=1, \forall i \in I$.

Remark 1. A strong separable there follows weakly separable. From weakly separable there follows orthogonal but not vice versa (see [1]-[4]).

Remark 2. In the general theory of statistical decisions there often arises a problem of transition from a weakly separated family of probability measures to the corresponding strongly separated family. In 1981, A. Skozokhod (see [1]) proved (in $Z F C \& C H$ theory) proved that if the Continuum Hypothesis is true, then an arbitrary weakly separated family of probability measures, whose cardinality is not greater than the cardinality of the continuum is strongly separable.The validity of the inverse relation was established in (see [9]-[10]). In particular, It was shown there that if an arbitrary weakly separated family of probability measures whose cardinality is less than or equal to the cardinality of the continuum is strongly separated, then the continuum Hypothesis ( $\mathrm{CH}$ ) is true Applying Martin's axiom (MA) in $1984 \mathrm{Z}$. Zerekidze(see [2]-[4]) proved that an arbitrary weakly separated family of Borel probability measures defined in a separable completely metrizable space (i. e. Polish space) is strongly separated if its cordinality is not prated than the cardinality of the continuum. G. Pantsulaia proved (see [9]-[10]) this result is extended to all complete metric spaces whose topological weights are not measurable in a wider sence. Z. Zerekidze proved in ZF theory (see [8]) for the statistical structure $\left\{E, S, \mu_{i}, i \in I\right\}$ where $\mathrm{N}$ set at natural numbers, orthogonality, weak separability, separability and strong separability equivalent concepts.

Let I be set of parameters and B (I) $\sigma$-algebra of subsets of I which contains all finite subsets I. 
Definite 5. A statistical structure $\left\{E, S, \mu_{i}, i \in I\right\}$ will be said to admit a consistent estimators of parameter $i \in I$ if there exists at least one measurable map $\delta:(E, S) \rightarrow(I, B(I))$ such that $\bar{\mu}_{i}\{(x: \delta(x))=i\}=1, \quad \forall i \in I$.

We denote by $\bar{\mu}_{i}$ the completion of the measure $\mu_{i}$ and dom $\bar{\mu}_{i}$ the $\sigma$-algebra of all $\bar{\mu}_{i}$ measureable subsets of $E$

Remark 3. By A. Skorokhod was introduced definition a consistent estimators of parameters (see [1]).

Definite 6. A statistical structure $\left\{E, S, \mu_{i}, i \in I\right\}$ will be said to admit a consistent estimators of any parametric function if for any real bounded measurable function $g:(I, B(I)) \rightarrow R$ there exists at least one measurable function $f:(E, S) \rightarrow R$ such that $\bar{\mu}_{i}\{x: f(x)=g(i)\}=1, \quad \forall i \in I$. .

Definition 7. A statistical structure $\left\{E, S, \mu_{i}, i \in I\right\}$ will be said to admit an unbiased estimators of any parametric function if for any real bounded measurable function $g:(I, B(I)) \rightarrow R$ there exists at least one measurable function $\beta:(E, S) \rightarrow R$, such that $\int_{B} \beta(x) \bar{\mu}_{i}(d x)=g(i), \quad \forall i \in I$.

Remark 4. If a statistical structure $\left\{E, S, \mu_{i}, i \in I\right\}$ admitting a consistent estimators of parameters $i \in I$ then this statistical structure $\left\{E, S, \mu_{i}, i \in I\right\}$ which admits a consistent estimators for any parametric function and a statistical structure which admits an unbiased estimators of any parametric function (see [6]-[8]).

Let $M^{\sigma}$ be a real linear space of all alternating finite measures on $S$.

Definition 8.A linear subset $M_{\beta} \subset M^{\sigma}$ is called a Banach space of measures if:

1) A norm can be defined on $M_{\beta}$ so that $M_{\beta}$ will be a Banach space with respect to this norm,and for any orthogonal measures $\mu, v \in M_{\beta}$, and real number $\lambda \neq 0$ the enequality $\|\mu+\lambda v\| \geq\|\mu\|$ is fulfilled;

2) If $\mu \in M_{B},|f(x)| \leq 1$ than $v_{f}(A)=\int_{A} f(x) \mu(d x) \in M_{B}$ and $\left\|v_{f}\right\| \leq\|\mu\|$;

3) If $v_{n} \in M_{B}, v_{n}>0, v_{n}(E)<+\infty, n=1,2, \ldots$ and $v_{n} \downarrow 0$ then for any linear functional $\ell^{*} \in M_{B}^{*} \lim _{n \rightarrow \infty} \ell^{*}\left(v_{n}\right)=0$, where $M_{B}^{*}$ conjugate to $M_{B}$ linear space.

The definition and construction of the Banach space of measures is studied Z.Zerekidze(see [7]).

Definition 9. Let $\mathrm{I}$ some set of indexes and $M_{B_{i}}$ Banach space $\forall i \in I$. We $\operatorname{set} M_{B}=\left\{\left\{X_{i}\right\}_{i \in I}, X_{i} \in M_{B_{i}}, \sum_{i \in I}\left\|X_{i}\right\|_{M_{B_{i}}}<\infty\right\}$.

Then the $M_{B}$ with norm $\left\|\left\{X_{i}\right\}_{i \in I}\right\|=\sum_{i \in I}\left\|X_{i}\right\|_{M_{B_{i}}}<\infty$ is the Banach space. It is called the direct sum of Banach spaces $M_{B_{i}}$ and denoted so $M_{B}=\underset{i \in I}{\oplus} M_{B_{i}}$.

By Z. Zerakidze was introduced definition and is studied construction the Banach space of measures (see [7]) The following theorem has also been proved in this paper (see [7]).

Theorem 1. Let $M_{B}$ be a Banach space of measures, then in $M_{B}$ there exists a family of pairwise orthogonal probability measures $M=\left\{\mu_{i}, i \in I\right\}$ such that $M_{B}=\oplus_{i \in I} M_{B_{i}}$, where $M_{B_{i}}$ is the Banach space of elements $v$ of the norm : $v(B)=\int_{B} f(x) \mu_{i}(d x) B \in S, \int_{E}|f(x)| \mu_{i}(d x)<\infty,\|v\|_{M_{B_{i}}}=\int_{E}|f(x)| \mu_{i}(d x)$.

Let $t=\left(t_{1}, t_{2}, \ldots, t_{n}\right) \in T$, where $\mathrm{T}$ be closed bounded subset of $R^{n}, t \in T, i \in I$ Gaussian real homogenous field on $\mathrm{T}$ with zero means $E\left[\xi_{i}(t)\right]=0, \forall i \in I$ and correlation function 
$E\left[\xi_{i}(t) \xi_{i}(S)\right]=R_{i}(t-S), t, S \in T, i \in I$. Let $\left\{\mu_{i}, i \in I\right\}$ be the corresponding probability measures given on $\mathrm{S}$ and $f_{i}(\lambda), \lambda \in R^{n}, \forall i \in I$ be spectral densities.

We be called the Fourier transformation of generation Fourier transformation.

Let $\int_{R^{n} R^{n}} \int_{f_{i}} \frac{\left.\tilde{b}_{i, j}(\lambda, \mu)\right|^{2}}{f_{i}(\mu)} d \lambda d \mu=+\infty \quad \forall i, j \in I$, where $\tilde{b}_{i, j}(\lambda, \mu), \lambda, \mu \in R^{n}, \forall i, j \in I$ the Generalization Fourier transformation of the following functions $b_{i, j}(S, t)=R_{i}(S, t)-R_{j}(S, t), \mathbb{S}, t \in T, \forall i, j \in I$.

Then the corresponding probability measures $\mu_{i}$ and $\mu_{j}$ are pairwise orthogonal $\forall i, j \in I$ (see [5]) and $\left\{E, S, \mu_{i}, i \in I\right\}$ are Gaussian orthogonal homogeneous fields statistical structures.Next, we consider $\mathrm{S}$ measurable $g_{i}(x), \forall i \in I$ function such that $\sum_{i \in I} \int_{E}\left|g_{i}(x)\right| \mu_{i}(d x)<+\infty$. Let $M_{B}$ the set measures defined by formula $\quad v(B)=\sum_{i \in I_{1}} \int_{B} g_{i}(x) \mu_{i}(d x), \quad$ where $\quad I_{1} \subset I \quad$ a countable subsets $\quad$ in $\quad \mathrm{I}$ and $\sum_{i \in I_{1}} \int_{E}\left|g_{i}(x)\right| \mu_{i}(d x)<+\infty$, define a norm on $M_{B}$ by formula $\|v\|=\sum_{i \in I} \int_{E}\left|g_{i}(x)\right| \mu_{i}(d x)$, then $M_{B}$ is a Banach space of measures and $M_{B}=\bigoplus_{i \in I} M_{B_{i}}$, where $M_{B_{i}}$ in Banach space of elements the form $v(B)=\sum_{i \in I_{1}} g_{i}(x) \mu_{i}(d x), B \in S, \int_{E}\left|g_{i}(x)\right| \mu_{i}(d x)<+\infty$, with the norm on $M_{B_{i}}\|v\|_{M_{B_{i}}}=\int_{E}\left|g_{i}(x)\right| \mu_{i}(d x)$.

It is also well known that in the (ZFC), (CH), (MA) theory there exists a weakly separable statistical structure which is not strongly separable.Here and in the equal we denote by (MA) the Martin's axiom (see [2]-[8]).

Theorem 2. Let $M_{B}=\bigoplus_{i \in I} M_{B_{i}}$ be a Banach space of measures, $\mathrm{E}$ be the complete separable metric space. $\mathrm{S}$ be the Borel $\sigma$-algebra in $\mathrm{E}$ and $c a r d I \leq c$, where $c$ denotes a power of continuum. Then in the theory $(\mathrm{ZFC}) \&(\mathrm{MA})$ the Gaussian Homogeneous fields orthogonal statistical structures $\left\{E, S, \mu_{i}, i \in I\right\}$ admits a consistent estimators of parameters $i \in I$ if and only if the correspondence $f \rightarrow l_{f}$ defined by the equality $\int_{E} f(x) \mu_{i}(d x)=l_{f}\left(\mu_{i}\right), \quad \forall \mu_{i} \in M_{B}$ is one-to-one. Here $l_{f}$ is a linear continuous functional on $M_{B}$, $f \in F\left(M_{B}\right)$. Denote by $F=F\left(M_{B}\right)$ the set of real functions $f$ for which $\int_{E} f(x) \mu_{i}(d x)$ is defined $\forall \mu_{i} \in M_{B}$.

Proof.Necessity. The existence of a consistent estimator $\delta:(E, S) \rightarrow(I, B(I))$ of the parameter $i \in I$ implies that $(\forall i)\left(i \in I \rightarrow \bar{\mu}_{i}(\{x: \delta(x)=i\})=1\right)$.

Setting $X_{i}=\{x: \delta(x)=i\}$ for $i \in I$, we get:

1) $\bar{\mu}_{i}\left(X_{i}\right)=\bar{\mu}_{i}(\{x: \delta(x)=1\})$ for $i \in I$;

2) $X_{i_{1}} \cap X_{i_{2}}=\varnothing$ for all different parameters $i_{1}$ and $i_{2}$ from I because $\left(X_{i_{1}}=\left\{x: \delta(x)=i_{1}\right\}\right) \cap\left(\left\{x: \delta(x)=i_{2}\right\}=X_{i_{2}}\right)=\varnothing ;$

3) $\bigcup_{i \in I} X_{i}=\{x: \delta(x) \in I\}=E$.

Therefore a statistical structure $\left\{E, S, \mu_{i}, i \in I\right\}$ is strongly separable, so there exist S-measurable sets $\left\{X_{i}\right\}, i \in I$ such that $\mu_{i}\left(X_{j}\right)=\left\{\begin{array}{l}1, \text { if }=j \\ 0, \text { if } \neq j\end{array}\right.$ 
We put the linear continuous functional $l_{X_{i}}$ into the correspondence to a function $I_{X_{i}}=F\left(M_{B}\right)$ by the formula: $\int_{E} I_{X_{i}}(x) \mu_{i}(d x)=l_{I_{X_{i}}}\left(\mu_{i}\right)=\left\|\mu_{i}\right\|_{M_{B_{i}}}$.

We put the linear continuous functional $l_{\bar{f}_{1}}$ into the correspondence to the function $\bar{f}_{1}(x)=f_{1}(x) I_{X_{i}}(x)$ Then for

$\mu_{i_{1}} \in M_{B}\left(\mu_{i}\right) \int_{E} \bar{f}_{1}(x) \mu_{i_{1}}(d x)=\int_{E} f_{1}(x) I_{X_{i}}(x) \mu_{i_{1}}(d x)=\int_{E} f(x) f_{1}(x) I_{X_{i}}(x) \mu_{i}(d x)=l_{\bar{f}_{1}}\left(\mu_{i_{1}}\right)=\left\|\mu_{i_{1}}\right\|_{M_{B_{i}}}$.

any

Let $\Sigma$ be the collection of extensions of functional satisfying the condition $l_{f} \leq p(x)$ on those subspaces where they are defined.

Let us introduce on $\Sigma$ a partial ordering having assumed $l_{f_{1}}<l_{f_{2}}$ if $l_{f_{2}}$ is defined on large set then $l_{f_{1}}$ and $l_{f_{1}}=l_{f_{2}}$ there where both of them are defined.

Let $\left\{l_{f_{i}}\right\}_{i \in I}$ be a linear ordered subset in $\Sigma$. Let $M_{B_{i}}$ be the subspace on which $l_{f_{i}}$ is defined. Define $l_{f}$ on $\bigcup_{i \in I} M_{B_{i}}$ having assumed $l_{f}(\mu)=l_{f_{i}}(\mu)$ if $\mu \in M_{B_{i}}$.

It is obvious, that $l_{f_{i}}<l_{f}$. Since any linearly ordered subset in $\Sigma$ has an upper bound by virtue of Chorn's lemma $\Sigma$ contains a maximal element $\lambda$ defined on some set $X^{\prime}$ satisfying the condition $\lambda(x) \leq p(x)$ for $x \in X^{\prime}$. But $X^{\prime}$ must coincide with the entire space $M_{B}$ because otherwise. We could extended $\lambda$ to a wider space by adding as above one more dimension. This contradicts the maximality of $\lambda$ hence $X^{\prime}=M_{B}$. Therefore the extension of the functional is defined everywhere.The extension of the functional is defined everywhere.

It we put the linear continuous functional $l_{f}$ into correspondence to the function $f(x)=\sum_{i \in I} g_{i}(x) I_{X_{i}}(x) \in F\left(M_{B}\right) \quad$ then $\quad$ obtain $\quad \int_{E} f(x) \mu(d x)=\|\mu\|=\sum_{i \in I}\left\|\mu_{i}\right\|_{M_{B_{i}}}, \quad$ where $\mu(B)=\sum_{i \in I} \int_{B} g_{i}(x) \mu_{i}(d x), \quad B \in S$

The necessity is proved.

Sufficiency. For $f \in F\left(M_{B}\right)_{\text {we }}$ define linear continuous functional $l_{f}$ by the equality $\int f(x) \mu(d x)=l_{f}(\mu)$. Denote $I_{f}$ a countable subset in I, for which $\int_{E} f(x) \mu_{i}(d x)=0$ for $\mathrm{i} \notin I_{f}$. Let us consider functional $l_{f_{i}}$ on $M_{B_{i}}$ to which there corresponds. Then for $\mu_{i_{1}}, \mu_{i_{2}} \in M_{B_{i_{1}}}$ have $\int_{E} f_{i_{1}}(x) \mu_{i_{2}}(d x)=l_{f_{i_{1}}}\left(\mu_{i_{2}}\right)=\int_{E} f_{1}(x) f_{2}(x) \mu_{i_{1}}(d x)=\int_{E} f_{i_{1}}(x) \mu_{i_{1}}(d x)$ therefore $f_{i_{1}}=f_{1}$ a.e. with respect measure $\mu_{i_{1}}$. Let $f_{i}>0$ a. e. with respect to the measure $\mu_{i}$ and $\int_{E} f_{i}(x) \mu_{i}(d x)<\infty, \mu_{i}(c)=\int_{c} f_{i}(x) \mu_{i}(d x)$, then $\int_{E} f_{i}(x) \mu_{j}(d x)=l_{f_{i}}\left(\mu_{j}\right)=0 \quad \forall j \neq i$.

Denote $C_{i}=\left\{x \mid f_{i}(x)>0\right\}$, then $\int_{E} f_{i}(x) \mu_{i}(d x)=0 \quad \forall j \neq 0$.

Hence it follows that $\mu_{j}\left(C_{i}\right)=0 \forall j \neq i$. On the other hand $\mu_{i}\left(E-C_{i}\right)=0$ therefore the statistical structure $\left\{E, S, \mu_{i}, i \in I\right\}$ is weekly separable. A Boral orthogonal family of probability measures $\left\{\mu_{i}, i \in I\right\}, \operatorname{cardI} \leq c$ is weakly separable. Represent $\left\{\mu_{i}, i \in I\right\}$ as an inductive sequence $\mu_{i}<w_{1}$ where $w_{1}$ denotes the first ordinal number of the power of the set I. 
Sense the statistical structure $\left\{E, S, \mu_{i}, i \in I\right\}$ is weakly separable, there exists a family S-measurable sets $\left\{X_{i}\right\}, i \in I$ such that the following relations are fulfilled: $\mu_{i}\left(X_{j}\right)=\left\{\begin{array}{l}1, \text { if } i=j \\ 0, \text { if } i \neq j\end{array}\right.$

For all $i \in\left[0, w_{1}\right)$ and $j \in\left[0, w_{1}\right)$

We define $w_{1}$ sequence of parts $Z_{i}$ of the space $\mathrm{E}$ so that the following relations are fulfilled:

1) $Z_{i}$ is Borel subset in $\mathrm{E}$ for all $i<w_{1}$;

2) $Z_{i} \subset X_{i}$ for all $i<w_{1}$;

3) $Z_{i} \cap Z_{j}=\varnothing$ for all $i<w_{1}, j<w_{1}, i \neq j$;

4) $\mu_{i}\left(Z_{i}\right)=1$ for all $i<w_{1}$.

Assume that $Z_{0}=X_{0}$. Let further the partial sequence $\left\{Z_{j}\right\}_{j<i}$ be already defined for $i<w_{1}$.

It is clear, that $\mu^{*}\left(\bigcup_{j<i} Z_{j}\right)=0$. Thus there exists a Borel subset $Y_{i}$ of the space $\mathrm{E}$ such that the following relations are valid: $\bigcup_{j<i} Z_{j} \subset Y_{i}$ and $\mu\left(Y_{i}\right)=0$. Assume $Z_{i}=X_{i}-Y_{i}$, thereby the $w_{1}$ sequence of $\left\{Z_{i}\right\}_{i<w_{1}}$ disjunctive measurable subsets of space $\mathrm{E}$ is constructed. Therefore $\mu_{i}\left(Z_{i}\right)=1$. For all $i<w_{1}$. A statistical structure $\left\{E, S, \mu_{i}, i \in I\right\}$ is strongly separable.

The statistical structure $\left\{E, S, \mu_{i}, i \in I\right\}$ is strongly separated there exists a family $\left(Z_{i}\right)_{i \in I}$ of elements of $\sigma$-algebra $S_{1}=\bigcap_{i \in I} \operatorname{dom}\left(\bar{\mu}_{i}\right)$ such that:

1. $\bar{\mu}_{i}\left(Z_{i}\right)=1, \forall i \in I$;

2. $Z_{i_{1}} \cap Z_{i_{2}}=\varnothing$ for all different parameters $i_{1}$ and $i_{2}$ from I;

3. $\bigcup_{i \in I} Z_{i}=E$.

For $x \in E$, we put $\delta(x)=i$, where i is unique parameter from the set I for which $x \in Z_{i}$. The existence of such a unique parameter I can be proved by using conditions (2), (3).

Now let $Y \in B(I)$. Then $\{x: \delta(x) \in Y\}=\bigcup_{i \in Y} Z_{i}$. We have to show that $\{x: \delta(x) \in Y\}=\operatorname{dom}\left(\bar{\mu}_{i_{0}}\right)$ for each $i_{0} \in I$.

If $i_{0} \in Y$ then $\{x: \delta(x) \in Y\}=\bigcup_{i \in Y} Z_{i}=Z_{i_{0}} \bigcup \bigcup_{i \in Y-i_{0}} Z_{i}$.

On the other hand, from the validity of the condition that the statistical structure $\left\{E, S, \mu_{i}, i \in I\right\}$ is strongly separated it follows that $Z_{i_{0}} \in S_{1}=\bigcap_{i \in I} \operatorname{dom}\left(\bar{\mu}_{i}\right) \subseteq \operatorname{dom}\left(\bar{\mu}_{i_{0}}\right)$.

On the other hand, the validity of the condition $\bigcup_{i \in Y-i_{0}} Z_{i} \subseteq\left(E-Z_{i_{0}}\right)$ implies that $\bar{\mu}_{i_{0}}\left(\bigcup_{i \in Y-i_{0}} Z_{i}\right)=0$. The latter equality yields that $\bigcup_{i \in Y-i_{0}} Z_{i} \in \operatorname{dom}\left(\bar{\mu}_{i_{0}}\right)$.

Since $\operatorname{dom}\left(\bar{\mu}_{i_{0}}\right)$ is $\sigma$-algebra, we deduce that $\{x: \delta(x) \in Y\}=Z_{i_{0}} \bigcup \bigcup_{i \in Y-i_{0}} Z_{i} \in \operatorname{dom}\left(\bar{\mu}_{i_{0}}\right)$. 
If $i_{0} \notin Y$, then $\{x: \delta(x) \in Y\}=\bigcup_{i \in Y} Z_{i} \subseteq\left(E-Z_{i_{0}}\right)$ and we claim that $\bar{\mu}_{i_{0}}\{x: \delta(x) \in Y\}=0$. The letter relation implies that $\{x: \delta(x) \in Y\} \in \operatorname{dom}\left(\bar{\mu}_{i_{0}}\right)$.

Thus we have shown the validity of the condition $\{x: \delta(x) \in Y\} \in \operatorname{dom}\left(\bar{\mu}_{i_{0}}\right)$ for an arbitrary $i_{0} \in I$. Hence $\{x: \delta(x) \in Y\} \in \bigcap_{i \in I} \operatorname{dom}\left(\bar{\mu}_{i_{0}}\right)=S_{1}$. We have shown that the map $\delta:(E, S) \rightarrow(I, B(I))$ is measurable map.

Since $B(I)$ contain all singletons of $\mathrm{I}$, we claim that $(\forall i)\left(i \in I \rightarrow \bar{\mu}_{i}(\{x: \delta(x)=i\})=\bar{\mu}_{i}\left(Z_{i}\right)=1\right)$.

Theorem 2 is proved.

Analogously is proved

Theorem3. Let $M_{B}=\oplus_{i \in I} M_{B_{i}}$ Be a Banach space of measures.E be the complete metric space, whose topological weights are not measurable in a wider sense. $\mathrm{S}$ be the Borel $\sigma$-algebra in $\mathrm{E}$ and $c a r d I \leq c$. Then in the theory (ZFC)\&(MA) the Gaussian homogeneous fields orthogonal statistical structures $\left\{E, S, \mu_{i}, i \in I\right\}$ admits a consistent estimators of parameters $i \in I$ and only if the correspondence $f \rightarrow l_{f}$ Defined by the equality $\int_{E} f(x) \mu_{j}(d x)=l_{f}\left(\mu_{i}\right) \forall \mu_{i} \in M_{B}$ is one-to-one. Here $l_{f}$ is linear continuous functional on $M_{B}, f \in F\left(M_{B}\right)$ Denote by $F=F\left(M_{B}\right)$ the set of real functions $\mathrm{f}$ for which $\int_{E} f(x) \mu_{j}(d x)$ is defined $\forall \mu_{i} \in M_{B}$.

\section{References}

[1]. Ibramhalilov A. Skorokhod. Consistent estimates of parameters of random processes. Naukova Dumka. Kiev (1980)

[2]. T. Jech. Set Theory. Springer, Berlin (2003),

[3]. A. Kharazishvili Topological aspects of measure theory. Naukova Dumka. Kiev (1984)

[4]. Z. Zerekidze. On weakly divisible and divisible families of probability measure. Bull Acad.Sci.Georgian SSR 113(2) 273-275 (1984)

[5]. Z. Zerekidze. About the conditions equivalence of Gaussian measures corresponding to homogeneous fields. Works of Tbilisi State University. Vol. 2 215-220 (1969)

[6]. Z. Zerekidze . On consistent estimators for families of probability measures.In : 5-th Japan - USSR Symposium on Probability Theory. Kyoto, pp 62-63 (1986)

[7]. Z. Zerekidze. Banach space of measures In: Gzigelionics, B, at al (eds.) Probability Theory and Methematical Statistics :Proceedings of the Fifth Vilnius Conference, Vilnius,vol.2 VSP/Mokslas pp. 609-616 (1991)

[8]. Z. Zerekidze. M. Mumladze. Statistical structures and consistent criteria for checking hypotheses Lambert Academic Publishing. ISBN: 978-2-659-69689-3 Saarbrucken Deutschland 2015

[9]. G. Pantsulaia. On orthogonal families of probability measures (in Russian) Trans.GP1-8 (3501-pp 106-112) (1989)

[10]. G. Pantsulaia. On separation properties for families of probability measures. Georg Math. J 10(2) pp. 335-342 (2003) 